\title{
Quintessence with two energy scales
}

\author{
Philippe Brax* \\ CERN, Theory Division, Genève, Switzerland \\ Jérôme Martin ${ }^{\dagger}$ \\ Institut d'Astrophysique de Paris, 98 boulevard Arago, 75014 Paris, France \\ Alain Riazuelo \\ Département de Physique Théorique, Université de Genève, 24 Quai Ernest Ansermet 1211 Genève-4, Switzerland
}

(Received 26 April 2001; published 25 September 2001)

\begin{abstract}
We study quintessence models using low energy supergravity inspired from string theory. We consider effective supergravity with two scales $m_{S}$, the string scale, and $m_{P l}$, the Planck scale, and show that quintessence naturally arises from a supersymmetry breaking hidden sector. As long as supersymmetry is broken by the $F$-term of a Polonyi-like field coupled to the quintessence field in the Kähler potential we find that the Ratra-Peebles potential and its supergravity version are generic predictions. This requires that the string scale decouples from the Planck scale, $m_{S} \ll m_{P l}$. In the context of supergravity, the potential possesses a minimum induced by the supergravity corrections to the Ratra-Peebles potential at low redshifts. We study the physical consequences of the presence of this minimum.
\end{abstract}

DOI: 10.1103/PhysRevD.64.083505

PACS number(s): 98.80.Cq, 04.65.+e

\section{INTRODUCTION}

The cosmological constant problem is a long standing problem in theoretical physics [1]. Although it first appeared in the context of general relativity, it is now clear that this problem is also deeply rooted in high energy physics. There exist several facts which explain this link. The first is the observation that the zero-point energy of quantum fields living in our universe effectively acts as a bare cosmological constant of the Einstein equations. The total contribution that we measure is therefore a combination of these two terms. Since a naive argument leads to a zero-point energy comparable to the Planck energy, and since astrophysical observations tell us that the contribution is of the order of the critical energy density, an extraordinary cancellation is needed if one wishes to reconcile experiments with theories. A second fact became more evident after the advent of supersymmetry (SUSY) [2]. Indeed, in global SUSY, the zero-point energy is guaranteed to vanish [3]. This raises the hope of finding a mechanism where the cosmological constant would be exactly zero. However, this explanation fails because SUSY has to be broken in order to explain the heavy masses of the superpartners. This means that the cosmological constant has to be at least of the order of the SUSY breaking scale, i.e., at least of order $1 \mathrm{TeV}$, a value which still requires a very accurate fine-tuning. On the other hand, when gravity is taken into account, leading to supergravity (SUGRA), the fundamental state does not necessarily have a zero energy. In that case, when SUSY is broken, there is still the hope of finding a vanishing result. With the present state of the art, this requires again a fine-tuning, and cannot be derived from a

\footnotetext{
*Email address: Philippe.Brax@cern.ch

†Email address: jmartin@iap.fr

‡Email address: Alain.Riazuelo@obspm.fr
}

fundamental principle. Therefore, the very small (vanishing?) value of the cosmological constant remains a mystery. The previous considerations led many people to believe that its value could be understood in the framework of the most promising theory of high energy that was nowadays at disposal: string theory [4]. It is widely believed that the only natural outcome of string theory is that it must be zero [5]. However, it is clear that we are far from being in a situation where this theoretical prejudice can be convincingly justified.

An explanation for this puzzling question has recently become even more necessary, since a combination of astrophysical observations, including, among others, measurements of the Hubble diagram with type Ia supernovae [6], seem to indicate that a form of dark energy now dominates our universe; also see Refs. [7-13]. This has led to the idea of quintessence. In this framework, the cosmological constant vanishes exactly, due to an as yet unknown mechanism, and a scalar field is responsible for the acceleration of the expansion of the universe [14-18]. In the same manner as for the vanishing cosmological constant, it seems likely that the physical nature of this field can be understood within the framework of string theory, or at least within a framework of theories describing its low energy limit. In this paper, we will adopt this point of view.

Let us now recall some of the main ingredients of an effective SUGRA description of string theory. The effective action describes the low energy degrees of freedom which can be viewed as massless string excitations. By computing string scattering amplitudes, one can build an order by order perturbative expansion in the massless fields. This perturbative expansion possesses two characteristic scales: the string scale $m_{S}$, and the compactification radius $R_{c}$ springing from the necessary compactification from ten to four dimensions. These two scales can be combined to form the Planck scale $m_{P l}$ which naturally appears at string tree level and parametrizes the SUGRA expansion. The effective Lagrangian ap- 
pears as a double series in the string scale and the Planck scale. In the context of heterotic theory, both these scales are large and almost coincide. In new scenarios involving type I strings and $D$ branes there can be a decoupling regime $m_{S}$ $\ll m_{P l}[19]$. We will show that, in this context, the SUGRA potential introduced in Refs. [20,21] is a prediction of the theory (for another SUGRA model of quintessence, see Ref. [22]).

It is also of the utmost importance to understand which role SUSY breaking plays in the determination of the shape of the quintessence potential. As already mentioned above, particle physics experiments require that SUSY must be broken at a scale at least of the order of $1 \mathrm{TeV}$. This is notoriously difficult to achieve in explicit string models. Indeed, such a breaking must arise from nonperturbative effects which are often difficult to control. Despite the absence of convincing models of SUSY breaking, one can use a more phenomenological approach and parametrize the SUSY breaking sector by $F$ terms responsible for the breaking [23]. This is the approach we will follow. In particular, one usually assumes that SUSY is broken by $F$ terms of the dilaton $S$ and the moduli fields $T^{i}$ measuring the compactification scales. In new type I models one can also consider the blowup modes associated with fixed points of orbifolds [24]. This breaking is supposed to occur in a hidden sector only gravitationally coupled to the visible sector. At the SUGRA level one generally assumes that the cosmological constant vanishes, requiring that $(S+\bar{S})^{2}\left|F_{S}\right|^{2}+\sum_{i=1}^{3}\left(T^{i}+\bar{T}^{i}\right)^{2}\left|F_{T^{i}}\right|^{2}$ $=3 m_{3 / 2}^{2} / \kappa$, where $m_{3 / 2}$ is the gravitino mass. This relation is imposed in order to cancel large contributions when the breaking scale is of order of the a few $\mathrm{TeV}$, a mere 60 orders of magnitude larger than the critical density of the universe. In the context of quintessence we will reconsider the previous relation and analyze contributions which lead to quintessential potentials. In particular, and in order to comply with the existence of an attractor for the quintessence field, we will have to consider that quintessence arises from a hidden sector. This guarantees that the very small mass of the quintessence field does not lead to the existence of a long-range fifth force [25]. Moreover, we will see that quintessence can be most easily achieved in a SUSY breaking sector. In order to guarantee that the sparticle masses do not depend strongly on the quintessence field and therefore on the evolution of the universe, we find that the hidden sectors responsible for quintessence and the superpartner masses must differ.

This paper is organized as follows. In Sec. II, we quickly review how the effective SUGRA inspired from string theory can be used to calculate the shape of the quintessence potential. The details could have been dropped in a paper intended for high energy physicists, but we think that they are useful in order to render this paper self-consistent for a more general audience. In Sec. III, we study the SUGRA model proposed in Ref. [20] with $m_{S} \simeq m_{P l}$, which leads to the socalled Ratra-Peebles potential [14] of Eq. (1). In particular, we study the corrections to this model, and show that observable quantities like the equation of state parameter and its derivative are sensitive to these corrections. In Sec. IV, we study the generic shape of a potential arising from effective
SUGRA where the assumption $m_{S} \simeq m_{P l}$ has been relaxed. We prove that, at small redshifts, a generic form of the potential is precisely the one found in Refs. [20,21]. In addition, it is also established that the corrections no longer modify the shape of the potential, which now really appears as a prediction and not as the result of a particular model. In Sec. V, we study how SUSY breaking by moduli fields can affect the form of the potential. Again, it is found that the generic prediction is not changed. Finally, since the SUGRA potentials generically possess a minimum, in Sec. VI we study the observational consequences of this fact. It is demonstrated that the quintessence field oscillates at the bottom of its potential, but, depending on the precise depth of this minimum, the field may or may not have begun its oscillations today. It is also shown that in this framework it is unlikely that the minimum of this potential can be put to zero.

\section{MODEL BUILDING AND EFFECTIVE SUPERGRAVITY}

One of the main advantages of the quintessence scenario is that the coincidence problem can be solved, i.e., it is not necessary to fine-tune the initial conditions at reheating in order to understand why the dark energy starts dominating the matter content of the Universe nowadays. This is due to the fact that for a potential with the shape [14]

$$
V(Q)=\frac{M^{4+\alpha}}{Q^{\alpha}}
$$

(typically), the Klein-Gordon equation possesses a (scaling) solution [26] which is an attractor, also referred as a "tracking solution" [17]. This means that, whatever the initial conditions are, in an allowed range encompassing more than 100 orders of magnitude, a given solution of the Klein-Gordon equation will always tend toward the attractor before the present epoch. When the field is on tracks, it satisfies the equation $[14,17]$

$$
\frac{d^{2} V(Q)}{d Q^{2}}=\frac{9}{2} H^{2} \frac{\alpha+1}{\alpha}\left(1-\omega_{Q}^{2}\right),
$$

where $H$ is the Hubble parameter, and $\omega_{Q}$ is the equation of state parameter, i.e., the pressure to energy density ratio of the scalar field. This is an important equation, because it allows to understand the different regimes undergone by the quintessence field during cosmic evolution. Therefore, it can be used as a hint to which kind of physics must be used in order to build a realistic and successful model of quintessence. Equation (2) has the following consequences. First, it implies that the mass of the quintessence field now is of the order of $H_{0} \simeq 10^{-43} \mathrm{GeV}$. Such a small mass entails that direct couplings between the quintessence field and standard model fields have to be extremely suppressed. This suggests that the quintessence field belongs to a hidden sector of the theory in order to avoid direct couplings with the standard model fields, which would result in the existence of a nonobserved long-range interaction. Second, since the second derivative of the potential is approximatively given by 
$\simeq \rho_{Q} / Q^{2}$ and since, when the field is about to dominate, we also have $H^{2} \simeq \rho_{Q} / m_{P l}^{2}$, we deduce that, at small redshift, $Q \simeq m_{P l}$. This means that supergravity effects will be important at small redshifts, for example for the calculation of the numerical value of the equation of state. In addition, we can also estimate the value of the scale $M$ in the potential. One has

$$
M \simeq\left(\rho_{c} m_{P l}^{\alpha}\right)^{1 /(4+\alpha)},
$$

where $\rho_{c}$ is the critical energy density. Third, we know that, initially, the value of the energy density of the dark energy must be between the value of the background energy density at reheating, i.e., $\rho_{\text {reh }} \simeq 10^{61} \mathrm{GeV}^{4}$, and the background energy density today, i.e., $\rho_{c} \simeq 10^{-47} \mathrm{GeV}^{4}$. Starting from this range guarantees that the field $Q$ will join the attractor before now. This range for the initial energy density of the dark energy corresponds to very small values of the field itself, $Q \ll m_{P l}$. More precisely, if the field starts at rest, we initially have $10^{-108 / \alpha} m_{P l} \leqslant Q \leqslant m_{P l}$. Unless the field starts with an energy density of the same order as today's critical density, this implies that supergravity effects are negligible at the beginning of the evolution, and that this epoch can be well described by means of a globally supersymmetric theory.

Having identified the orders of magnitude of the value of the scalar field throughout the cosmic evolution, we can study the physics which is necessary to describe these different regimes. We are going to consider an effective SUGRA theory and the constraints imposed by quintessence. In particular, as mentioned above, we assume explicitly that the quintessence field belongs to a hidden sector of the theory. We assume that the effective action is a double series expansion in the Planck mass and in the string scale. The Planck mass $m_{P l}$ and the string scale $m_{S}$ are a priori two independent scales. The only experimental constraint is that $m_{S}$ $>1 \mathrm{TeV}$ in order not to be in conflict with the measurements performed by the accelerators. In heterotic string inspired models it was often assumed that $m_{S} \simeq m_{P l}$, because of the constraints on the perturbative unification scale. However, recently, models where the string scale is much lower than the Planck scale were proposed [19]. Generically, these two scales are linked by the equation

$$
m_{P l}^{2}=m_{S}^{8} V_{6},
$$

where $V_{6}$ is the volume of the six compactified dimensions. The constraint that $m_{S}>1 \mathrm{TeV}$ translates into a constraint on the volume $V_{6}<10^{14} \mathrm{GeV}^{-6}$. As mentioned above, it was recently shown that some of the compactified dimensions can be large (in comparison to the Planck length) resulting in a string scale much lower than the Planck scale. In this paper, for the moment, we leave $m_{P l}$ and $m_{S}$ free. We will discuss the different cases later on.

Because of the large value $Q \simeq m_{P l}$ of the quintessence field today, it appears that one would need a full understanding of the complete SUGRA action, i.e., one would need to take into account all $Q / m_{P l}$, and $Q / m_{S}$ terms in the Lagrangian. As a result, one would expect that an appropriate description of quintessence requires an understanding of nonperturbative effects either at the field theoretical level or even at the string level. In the following we shall use a more modest approach, and remain within a perturbative setting where the Lagrangian is expanded in inverse mass powers. We will pay particular attention to the sensitivity of the physical observables to the degree of the truncated perturbative series. In particular we will comment on the stability of the physical observables under a change of the truncation degree. The only nonperturbative inputs will be the SUSY breaking parameters.

Let us first consider the early universe evolution of the quintessence field. Setting the initial conditions at reheating, after inflation, implies that for most of the time the quintessence field takes values which are negligible with respect to the Planck mass. We assume that the expectation values of the other fields are also negligible in comparison with the Planck mass. This means that, in this context, the most general Lagrangian is given by the $N=1$ (global) SUSY Lagrangian [i.e., the $N=1$ SUGRA Lagrangian where terms of order $O\left(m_{P l}^{-1}\right)$ are neglected]

$$
\begin{aligned}
\mathcal{L}= & \int d^{4} \theta K\left(\Phi^{i k \dagger} e^{2 g_{k} V_{k}}, \Phi^{i k}\right)+\int d^{2} \theta\left[W\left(\Phi^{i k}\right)+\text { H.c. }\right] \\
& +\sum_{k} \int d^{2} \theta \sum_{a b} f_{a b}\left(\Phi^{i l}\right)\left[W_{k a}{ }^{\alpha} W_{k b \alpha}+\text { H.c. }\right] \\
& +\sum_{k \in U(1)} \xi_{k} \int d^{4} \theta V_{k} .
\end{aligned}
$$

Because of the possible large hierarchy between the string scale and the Planck scale, the Kähler potential $K$ and the superpotential $W$ are now series in the inverse string scale. Let us now focus on the hidden sector containing the quintessence field. For simplicity, and since it does not change our general argument, we will take $f_{a b}\left(\Phi^{i l}\right)=\delta_{a b}$. Let us describe this Lagrangian in more detail. In the previous expression, $\Phi^{i k}\left(x^{\kappa}, \theta, \bar{\theta}\right)$ is a chiral superfield and $V_{k}\left(x^{\kappa}, \theta, \bar{\theta}\right)$ is a vector superfield which can be written in terms of components as

$$
\begin{aligned}
\Phi^{i k}\left(x^{\kappa}, \theta, \bar{\theta}\right)= & \varphi^{i k}\left(x^{\kappa}\right)+\sqrt{2} \theta \psi^{i k}\left(x^{\kappa}\right)+\theta^{2} F^{i k}\left(x^{\kappa}\right), \\
V_{k}\left(x^{\kappa}, \theta, \bar{\theta}\right)= & \sum_{a}\left[-\theta \sigma^{\mu} \bar{\theta} V_{k a \mu}\left(x^{\kappa}\right)+i \theta \theta \bar{\theta} \bar{\lambda}_{k a}\left(x^{\kappa}\right)\right. \\
& \left.-i \bar{\theta} \bar{\theta} \theta \lambda_{k a}\left(x^{\kappa}\right)+\frac{1}{2} \theta \theta \bar{\theta} \bar{\theta} D_{k a}\left(x^{\kappa}\right)\right] T_{a k} \\
\equiv & \sum_{a} V_{k a} T_{a k},
\end{aligned}
$$

where the vector superfield has been written in the WessZumino gauge. We assume that the above Lagrangian is invariant under the gauge group $G$ acting on the $k$ indices of the chiral superfields:

$$
G=\prod_{k} G_{k} \times U(1)_{X}
$$


This gauge group might become strongly coupled and lead to SUSY breaking via gaugino condensation. In the previous expressions, $k$ is a group index, i.e., $V_{k}$ is the superfield charged under the group $G_{k}$. Under this group $G_{k}$, many chiral superfields can be charged. The index $i$ in $\Phi^{i k}$ labels the different superfields that are charged under the group labeled by the index $k$. The matrices $T_{a k}$ are the generator of the gauge group $G_{k}$ and the index $a$ runs from 1 to $\operatorname{dim}\left(G_{k}\right)$. In the third term of the above Lagrangian, $W_{k a \alpha}$ is given by $W_{k a \alpha} \equiv-(1 / 4) \bar{D} \bar{D} e^{-V_{k a}} D_{\alpha} e^{V_{k a}}$, where $D$ is the supersymmetric derivative. The extra $U(1)_{X}$ is an anomalous Abelian factor associated with a Fayet-Iliopoulos term in the Lagrangian in order to cancel the anomaly by the GreenSchwarz mechanism [28]. In heterotic string theory there is a single anomalous $U(1)_{X}$ [29]. In type I string theories there may be several anomalous $U(1)$ 's depending on the geometry of the compactifying manifold [24]. The last term in the Lagrangian represents the Fayet-Iliopoulos term where $\xi_{k}$ is a constant different for each group, provided that this group is $U(1)$. A priori, the scale given by the Fayet-Iliopoulos term is expected to be of the order of the string scale. This is the case in the heterotic string theory for the unique FayetIliopoulos term for the anomalous $U(1)_{X}$. In type I string theories the Fayet-Iliopoulos terms are associated with a blowing up moduli of orbifold singularities in the compactification space. Their values parametrize a flat direction with no potential, and are therefore left unfixed at the perturbative level of string theory.

Once the Kähler function $K$ and the superpotential $W$ of the hidden sector are given, the Lagrangian [Eq. (5)] is completely fixed. In particular, the scalar potential can be calculated. It contains two contributions: one comes from the $F$ terms and the other comes from the $D$ terms. Explicitly, the potential is given by

$$
V=V_{F}+V_{D}=K_{\bar{A} B} F^{\bar{A} \dagger} F^{B}+\frac{1}{2} \sum_{k a} D_{k a} D_{k a}
$$

in the low energy limit. In the previous expression, we introduced a collective index $A \equiv(i k)$. The metric $K_{\bar{A} B}$ and the field $F_{A}$ can be expressed as

$$
K_{\bar{A} B}=\frac{\partial^{2} K}{\partial \varphi^{A \dagger} \partial \varphi^{B}}, \quad F^{A}=-K^{\bar{B} A} \frac{\partial W^{\dagger}}{\partial \varphi^{\bar{B} \dagger}},
$$

respectively, and the $D$ term is given by

$$
D_{k a}=-\frac{\xi_{k}}{2}-\sum_{i} g_{k} \frac{\partial K}{\partial \varphi^{i k \dagger}} T_{k a} \varphi^{i k \dagger}
$$

In the last equation, we have assumed that the gauge group considered is $U(1)$; otherwise the expression would be the same except that the Fayet-Iliopoulos term would not be present.

We now assume that SUSY is not broken by the $D$ terms. This implies that $\left\langle D_{k a}\right\rangle=0$. If $k$ corresponds to a group which is $U(1)$, this means that one (or many) of the scalar fields acquire a nonvanishing vacuum expectation value, according to

$$
\xi_{k}=-2 \sum_{i} g_{k}\left(\frac{\partial K}{\partial \varphi^{i k \dagger}} T_{k a} \varphi^{i k \dagger}\right), \quad k \in U(1) .
$$

The generators $T_{k a}$ give the charges of the fields under the considered $U(1)$. Typically, one expects $\left\langle\varphi^{i k}\right\rangle \simeq \sqrt{\xi_{k}}$. This means that the $U(1)$ gauge symmetries are broken at that scale. In the heterotic case this fixes the breaking at the Grand Unification Theory scale, while in the type I models the breaking scale is not specified as it is a modulus. We conclude that the $D$ part of the scalar potential vanishes, i.e.,

$$
V_{D}=0 \text {. }
$$

The nonzero contributions to the potential come from the $F$ terms.

The previous considerations are valid at very high redshift. However, at small redshift, one needs to take into account the effects of SUGRA, since the values of the quintessence field are not negligible compared to the Planck mass. In SUGRA, the form of the scalar potential is modified, and reads

$$
V=\frac{1}{\kappa^{2}} e^{G}\left(G^{A} G_{A}-3\right)+V_{D},
$$

where $\kappa=8 \pi / m_{P l}^{2}$ and $G \equiv \kappa K+\ln \left(\kappa^{3}|W|^{2}\right)$. In the previous expression, $G_{A}$ is defined by

$$
\begin{aligned}
& G_{A} \equiv \frac{\partial G}{\partial \varphi^{A}}=\kappa \frac{\partial K}{\partial \varphi^{A}}+\frac{1}{W} \frac{\partial W}{\partial \varphi^{A}}, \\
& G_{\bar{A}} \equiv \frac{\partial G}{\partial \varphi^{\bar{A} \dagger}}=\kappa \frac{\partial K}{\partial \varphi^{\bar{A} \dagger}}+\frac{1}{W^{\dagger}} \frac{\partial W^{\dagger}}{\partial \varphi^{\bar{A} \dagger}},
\end{aligned}
$$

and the indices are raised and lowered with the help of the following metric:

$$
G_{\bar{A} B} \equiv \frac{\partial^{2} G}{\partial \varphi^{\bar{A} \dagger} \partial \varphi^{B}}=\kappa K_{\bar{A} B} .
$$

The other terms in $G$ cancel out because the superpotential is a holomorphic function. A priori, this potential is no longer positive definite. In particular, there is a negative contribution coming from the superpotential.

Let us come to grips with the quintessence potential more precisely. According to the previous discussion, we only focus our attention to the $F$ part of the scalar potential. A first attempt to derive the Ratra-Peebles potential [Eq. (1)] from first principles was made in Ref. [27] and then in Refs. $[20,21]$. In order to see clearly the difference between this approach and the approach advocated in the present paper, we first quickly review the results obtained in Refs. [20,21]. Then we will study in detail new properties of the model presented in Ref. [21]. We will argue that these new proper- 
ties are in fact a problem, and we will see how, generically, they can be avoided in the context of theories with two different scales.

\section{A SUGRA MODEL LEADING TO THE RATRA-PEEBLES POTENTIAL}

In the model presented in Refs. [20,21], it is assumed that $m_{S} \simeq m_{P l}$. Contrary to the strategy used in Sec. IV, which is to see which kind of potential is obtained from a generic theory, the idea utilized in Refs. [20,21] was to study the required properties of the theory such that the desired potential (typically the Ratra-Peebles potential) is the result of the calculations described above. Below, we improve the presentation of the model of Refs. [20,21], in particular we describe it in a language closer to high energy physics than the one used in Refs. [20,21].

We assume that there are three sectors in the theory. One of them is the observable sector where all the known particles and their superpartners live and the two other sectors are hidden. The first hidden sector is the "quintessence sector" already mentioned above, where the quintessence field lives. The second one is the "broken sector," introduced such that SUSY should be broken in a satisfactory manner. We have seen previously that, generically, due to the presence of a Fayet-Iliopoulos term and to the vanishing of the potential coming form the $D$ terms, at least one scalar field acquires a nonvanishing vacuum expectation value. Let us call this field $Z$. Thus we have $\langle Z\rangle \neq 0$. This field belongs to the quintessence sector. In addition, this sector is required to contain a field $Y$ such that $\left\langle\partial_{Y} W\right\rangle \neq 0$. This field is similar to a Polonyi field [30], although we do not assume that the superpotential is linear in this field. We also assume that $\partial_{Y} W$, i.e., $F_{Y}$ in global SUSY, does not depend on $Q$. The Kähler potential and the superpotential of Refs. [20,21] have the forms

$$
\begin{aligned}
& K=\frac{1}{m_{P l}^{2 p}}|Y|^{2}(Q \bar{Q})^{p}+\hat{K}\left(|Y|^{4}, \ldots, \Phi_{Q}, \Phi_{\text {bro }}, \Phi_{\mathrm{obs}}\right), \\
& W=Y Z^{2}+\hat{W}_{Q}\left(\Phi_{Q}\right)+W_{\mathrm{bro}}\left(\Phi_{\mathrm{bro}}\right)+W_{\mathrm{obs}}\left(\Phi_{\mathrm{obs}}\right),
\end{aligned}
$$

where $\Phi_{Q}, \Phi_{\text {bro }}$, and $\Phi_{\text {obs }}$ denote superfields in the quintessence, broken, and observable sectors respectively. $W_{\text {bro }}$ and $W_{\text {obs }}$ are the superpotentials in the broken and observables sectors. $W_{Q} \equiv Y Z^{2}+\hat{W}\left(\Phi_{Q}\right)$ is the superpotential in the quintessence sector. We have $\left\langle W_{Q}\right\rangle=\left\langle W_{\text {obs }}\right\rangle=0$ but $\left\langle W_{\text {bro }}\right\rangle$ $\neq 0$. The condition $\left\langle W_{Q}\right\rangle=0$ guarantees that the SUGRA quintessence potential is positive definite. Then, in the context of global SUSY, the scalar potential is $V(Q)$ $=m_{P l}^{2 p}\left|F_{Y}\right|^{2} / Q^{2 p}$, i.e., the Ratra-Peebles potential. We see that a crucial point in the argument is the vanishing of the term $|Y|^{2}$ in the series defining the Kähler potential. Although this concerns only one term in the complete series, this should probably be considered as an unwanted finetuning, since there is no fundamental reason to expect that this term must be absent in a generic theory. In addition, since $M^{4+\alpha} \simeq \rho_{c} m_{P l}^{\alpha}$, one has

$$
\left|F_{Y}\right|^{2}=\left\langle Z^{2}\right\rangle^{2} \simeq \rho_{c},
$$

which fixes the scale at which SUSY is broken in the quintessence sector. We see that this scale is very small in comparison with the "natural scale" of SUSY breaking, i.e., $\simeq 1 \mathrm{TeV}$.

Actually, this is the main motivation for introducing two hidden sectors. It is convenient to break SUSY in a hidden sector since, from a phenomenological point of view, it seems difficult to break SUSY in the observable sector. Indeed, for example, a spontaneous breaking mechanism in the observable sector like the O'Raifeartaigh mechanism [31] would not lead to a spectrum in accordance with the constraints on the masses of the superpartners. Conversely, if the hidden sector contains a Polonyi field $P$ (not the same as the one contained in the quintessence sector, see above) such that $\left\langle F_{P}\right\rangle=m_{S B}^{2}$ and if the cosmological constant problem is assumed to be solved (as it is always the case when one discusses quintessence, see the introduction) then $m_{S B}^{2}$ $\simeq m_{3 / 2} m_{P l}$, where $m_{3 / 2}$ is the gravitino mass. This will give a mass of order $m_{3 / 2}$ to the superpartners. Since we expect $m_{3 / 2} \simeq 1 \mathrm{TeV}$, this implies $m_{S B} \simeq 10^{10} \mathrm{GeV}$ and $\left\langle F_{P}\right\rangle$ $\simeq 10^{20} \mathrm{GeV}^{2}$, a value far from $F_{Y}$. Therefore, it is necessary that the observable sector should be different from the broken sector in order to have a correct spectrum, and it is also necessary that the quintessence sector should be different from the broken sector in order to have a value for $m_{S B}$ of the correct order of magnitude. In addition, the quintessence sector cannot be the observable sector, since this would imply the presence of a long range fifth force not seen in the data. In order to obtain the potential which is valid not only at the beginning of the evolution but everywhere, we need to insert the Kähler potential and superpotential given in Eqs. (18) and (19) in the equation giving the scalar potential in SUGRA [Eq. (14)]. We find that the only contributions which lead to non-vanishing terms in the scalar potential are

$$
G_{\bar{Y} Y}=\kappa K_{\bar{Y} Y}, \quad F_{Y} \equiv-\frac{\partial W}{\partial Y}-\kappa W K_{Y} \neq 0,
$$

where $W$ stands for the total superpotential. ${ }^{1}$ The vacuum expectation value of the last term is in fact just $F_{Y}=$ $-\partial W / \partial Y$. This is due to the vanishing of the Polonyi-like field $\langle Y\rangle=0$ and the quadratic dependence of the Kähler potential on $Y$. Finally, we arrive at a positive definite expression

$$
V=e^{\kappa K} K^{Y \bar{Y}}\left\langle Z^{2}\right\rangle^{2},
$$

where we have used the fact that the $D$ terms are not modified in SUGRA and that, as a consequence, $\left\langle V_{D}\right\rangle=0$. The main difference comes from the exponential factor which represents the SUGRA corrections. However, we do not have yet reached our main goal because the kinetic term of $Q$ is still nonstandard. Indeed, since we are now in a regime

\footnotetext{
${ }^{1}$ Throughout the paper the auxiliary $F$ fields are given by $\mathcal{F}$ $=e^{\kappa K / 2} F$, where $F$ is defined by the second equation of Eqs. (21).
} 
where $Q \simeq m_{P l}$, we can no longer neglect the higher order terms in Eqs. (18) and (19), and thus $K_{Q \bar{Q}} \neq 1$. The Kähler potential evaluated at the minimum of the potential for the matter fields reads

$$
\begin{aligned}
K\left(Q,\langle Y\rangle,\langle Z\rangle,\left\langle\varphi^{i k}\right\rangle\right) & =\hat{K}\left(Q,\langle Z\rangle,\left\langle\varphi^{i k}\right\rangle\right) \\
& =\sum_{n=1}^{\infty} \frac{c_{2 n}}{m_{P l}^{2(n-1)}} Q^{2 n},
\end{aligned}
$$

where we have fixed the other hidden sector fields to their vev. This means that the coefficients $c_{2 n}$ are functions of $\langle Z\rangle$. In a regime where $Q \ll m_{P l}$, only the first term will be important, and leads to a canonical kinetic term for quintessence (with $c_{2}=1$ ). Therefore, the potential obtained in the context of global SUSY is not modified by a redefinition of the field. Closer to the Planck scale the contributions from the other terms become non-negligible. To deal with this problem, we define a new scalar field $\widetilde{Q}$ such that

$$
\mathrm{d} \widetilde{Q}=\sqrt{2 K_{Q \bar{Q}}} \mathrm{~d} Q \Rightarrow \widetilde{Q}=\int \mathrm{d} Q \sqrt{2 K_{Q \bar{Q}}} \equiv f(Q),
$$

where the function $f(Q)$ has been obtained by quadrature. The field $\widetilde{Q}$ has a standard kinetic term. Expressing $Q$ $=f^{-1}(\widetilde{Q})$, we obtain the SUGRA potential

$$
V(\widetilde{Q})=e^{\kappa K\left[f^{-1}(\widetilde{Q})\right]} \frac{\left\langle Z^{2}\right\rangle^{2}}{\left[f^{-1}(\widetilde{Q})\right]^{2 p}} .
$$

A priori, any function $f(Q)$ is allowed. When $Q \ll m_{P l}$, the form of the function $f$ is irrelevant, since we know from the previous SUSY considerations that the potential will be of the form $V(Q) \propto Q^{-2 p}$. If the Kähler function is just given by $K=Q \bar{Q}$, then the kinetic terms are standard, and we recover the SUGRA quintessence potential already studied in Ref. [20]:

$$
V(\widetilde{Q})=e^{\kappa \widetilde{Q}^{2} / 2} \frac{\left\langle Z^{2}\right\rangle^{2} m_{P l}^{2 p}}{\widetilde{Q}^{2 p}} .
$$

The physical consequences of the SUGRA corrections are numerous, and the potential given by Eq. (26) was studied in detail in Refs. [20,21,32]. There, it was shown that these corrections lead to a better agreement with the currently available data. In particular, the equation of state parameter is now given by $\omega_{Q} \simeq-0.82$, a value closest to -1 than in the usual quintessence models. The calculation of the Cosmic Microwave Background (CMB) multipoles in presence of SUGRA quintessence also show that the theoretical predictions are consistent with the most recent data, in particular the MAXIMA-1 data [32,33]. On the other hand, it is clear that we have assumed that the $Q$ kinetic terms are canonical. If this hypothesis is not fulfilled, potential (26) is modified and we see that the form of the potential above strongly depends on the Kähler potential.

Let us study how the scalar potential is modified when more terms in the Kähler potential are taken into account. In

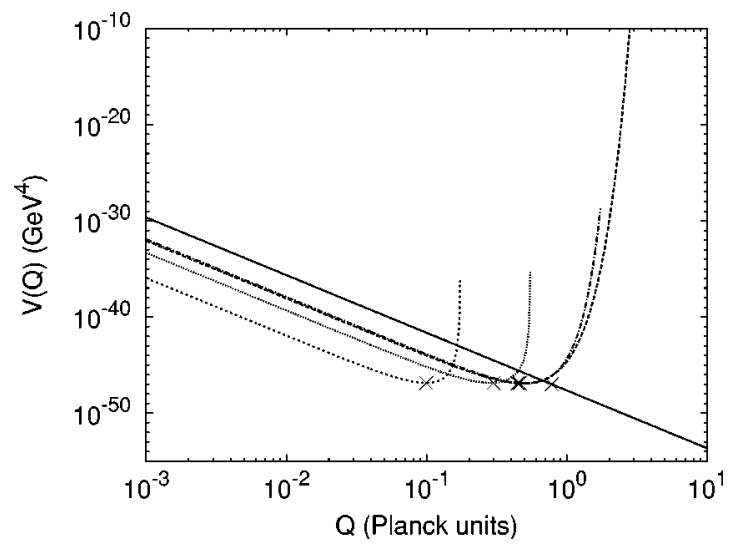

FIG. 1. Comparison between Ratra-Peebles and SUGRA potentials. The Ratra-Peebles potential [Eq. (1), solid line] is simply an inverse power law and always decreases. The standard SUGRA potential [Eq. (26), long-dashed line] possesses an exponential correction which dominates when the field takes values close to the Planck mass. The other SUGRA potential we have considered in Eq. (29) is plotted for $a=100$ (short-dashed line), $a=10$ (dotted line), and $a=-1$ (dot-dashed line). All the curves were plotted with $\alpha=2 p=6$ and normalized so that the quintessence field has a density parameter $\Omega_{Q}=0.7$ today, which roughly corresponds to put the minimum of the potential at $\rho_{\mathrm{c}}$. In addition, with crosses we have indicated the position of the quintessence field today. It is clear that the field has almost reached the minimum of its potential in all (SUGRA) cases.

particular, one would like to know whether the observables (for example, the equation of state parameter) are strongly dependent on the higher terms in series (23). Therefore, in order to have a more accurate description of the true Kähler potential, it is interesting to take into account one more term, and to choose

$$
K=|Q|^{2}+a \frac{|Q|^{4}}{m_{P l}^{2}},
$$

where $a$ is a new free parameter, leading to the following exact function $f(Q)$ :

$$
\begin{aligned}
\widetilde{Q}= & \frac{1}{\sqrt{2}}\left[Q \sqrt{1+4 a \frac{Q^{2}}{m_{P l}^{2}}}\right. \\
& \left.+\frac{m_{P l}}{2 \sqrt{a}} \ln \left(2 \sqrt{a} \frac{Q}{m_{P l}}+\sqrt{1+4 a \frac{Q^{2}}{m_{P l}^{2}}}\right)\right] .
\end{aligned}
$$

Unfortunately, this function cannot be inverted exactly. However for our purpose, it is sufficient to find the corrected potential at leading order in the expansion in $Q / m_{P l}$. One finds

$$
V(Q)=\frac{M^{4+2 p}}{\left(Q-a \frac{Q^{3}}{3 m_{P l}^{2}}\right)^{2 p}} \exp \left[\frac{\kappa}{2}\left(Q^{2}-a \frac{Q^{4}}{6 m_{P l}^{2}}\right)\right] .
$$

Some examples of this potential are plotted in Fig. 1. 
Let us now study how the corrections described above can affect the global evolution. In particular, as mentioned above, one would like to know whether observable quantities are significantly modified by the new terms that we have considered in the series defining the Kähler potential. An interesting way to distinguish between these various models observationally is to look at the behavior of the quintessence field equation of state. It can be shown [34] that, provided one knows both the matter density of the Universe as well as its curvature, one can both recover the quintessence equation of state parameter $\omega_{Q}$, as well as its derivative today by studying the luminosity distance vs redshift relation, for example with supernovae type Ia. The parameter $\omega_{Q}$ can be approximated at low redshift by

$$
\omega_{Q} \simeq \omega_{0}+z \omega_{1}
$$

and both $\omega_{0}$ and $\omega_{1}$ can be recovered, at least in principle, with good data. In the case described by Eq. (29), for positive values of $a$, the potential has a steeper part around $Q$ $=\sqrt{3 / a} m_{P l}$ because it diverges. Therefore the potential possesses a minimum before $Q=\sqrt{3 / a} m_{P l}$. As already stated, the field has reached the usual tracking regime at earlier time (which corresponds to small values of $Q$ ); therefore, it reaches its minimum sooner in the case of large $a$. As a consequence, the quintessence field behaves more rapidly as a cosmological constant than in the standard SUGRA case, and of course than in the Ratra-Peebles case. This can be seen explicitly by looking at the position of the quintessence field on its potential (see Fig. 1), or by plotting $\omega_{Q}$ and its derivative today as a function of $a$ (see Fig. 2). Note, however, that, strictly speaking, at the end of the evolution, all the terms in the expansion of $f^{-1}(Q)$ should be taken into account since $Q / m_{P l} \simeq 1$. Therefore, the present calculation can only give a hint of what happens when the corrections in the Kähler potential are fully considered. For negative values of $a$, the potential does not diverge but grow faster because of the higher argument of the exponential part. Therefore, as for the $a>0$ case, the minimum of the potential occurs at lower values of $Q$, and the field behaves more rapidly like a cosmological constant.

The main conclusions that we can draw from the previous analysis are the following. In the context of effective SUGRA, there exists a Kähler function and a superpotential which lead to a class of model described by Eq. (25). However, these models depend on specific assumptions for the superpotential and Kähler functions. If more generic terms are considered in the series defining the Kähler potential, then some sensitivity of the observables to the form of the Kähler potential within this class of models is found, but as long as the potential possesses a minimum around $Q$ $\simeq m_{P l}$, the main features of the SUGRA potential of Refs. $[20,21]$ are preserved. Having identified the main advantages and drawbacks of the approach followed in Refs. [20,21], we now turn to a different method where some of the previous shortcomings can be avoided.

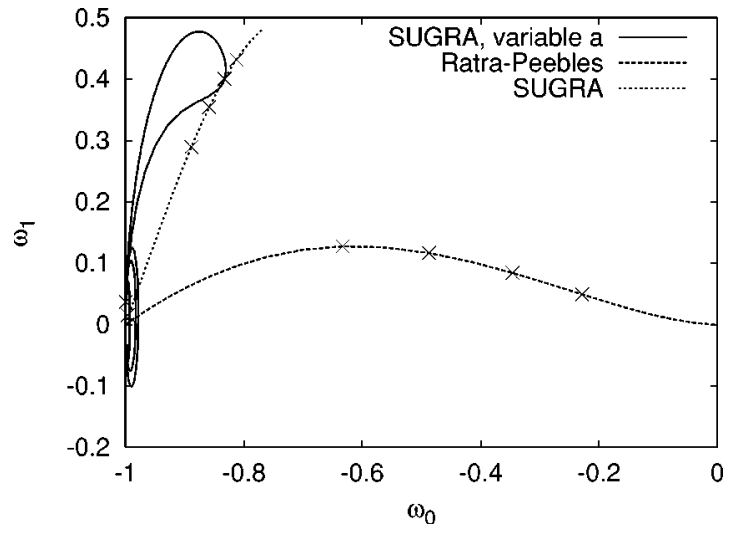

FIG. 2. Effect of a modification to the quintessence potential [Eq. (29)] on today's evolution of the quintessence equation of state (solid line). The three values of $a$ plotted in Fig. 1 are represented with crosses (the case $a=-1$ is near the intersection with the shortdashed line and the two others are near $\left.\omega_{0} \rightarrow-1, \omega_{1} \rightarrow 0\right)$. As explained in the text, almost any value of $a$ helps the quintessence field to mimic a cosmological constant $\left(\omega_{0} \rightarrow-1, \omega_{1} \rightarrow 0\right)$. In addition, we have plotted the dependence on $\alpha$ of the Ratra-Peebles (long-dashed line) and SUGRA (short-dashed line) potentials. For the two curves, $\alpha$ varies from $\ll 1$ (left) to $\gg 1$ (right). The fact that the field roughly behaves as a cosmological constant for low values of $\alpha$ comes from the fact that the potential is flatter and therefore the field stops more rapidly when it begins to dominate (even in the tracking regime, $1+\omega_{Q} \propto \alpha$; see Ref. [18]). Conversely, for high values of $\alpha$, the field tends to mimic the behavior of the background fluids. On these two curves, values of $\alpha=2,4,8$, and 16 are represented with crosses. The dependence on $\alpha$ of the SUGRA potential is much less important than in the Ratra-Peebles case.

\section{A GENERIC APPROACH TO QUINTESSENCE WITH TWO SCALES}

In this section, which constitutes the core of this paper, we adopt a different approach compared to that of Sec. III. Since a priori there is no reason to consider that $m_{S}$ and $m_{P l}$ are of the same order of magnitude, we do not make this artificial assumption. As a consequence, we consider that $m_{S}$ can have any value provided, of course, that it is smaller than the Planck mass, $m_{S} \ll m_{P l}$. Then, the strategy is as follows: instead of trying to find the Kähler potential and the superpotential which leads to the Ratra-Peebles potential as in Refs. [20,21], we will try to see which kind of potential arises from a generic Kähler potential and superpotential, i.e., without any fine-tuning of their shape. We still assume that there are three sectors in the theory, two of them being hidden. We first investigate this question in the context of global SUSY, i.e., when the value of the quintessence field is small in comparison to the Planck mass, which is the case just after reheating where the initial conditions are set. We assume that the Kähler potential is a nonsingular series as $Q$ goes to zero. Let us expand the Kähler potential focusing on the coupling between the quintessence field $Q$ and $Y$, the Polonyi field in the quintessence sector. One has

$$
K(Y, Q, \ldots)=|Y|^{2}+|Y|^{2} \sum_{p=1}^{p_{\max }} \frac{1}{m_{S}^{2 p}}(Q \bar{Q})^{p}+\hat{K}(\cdots),
$$




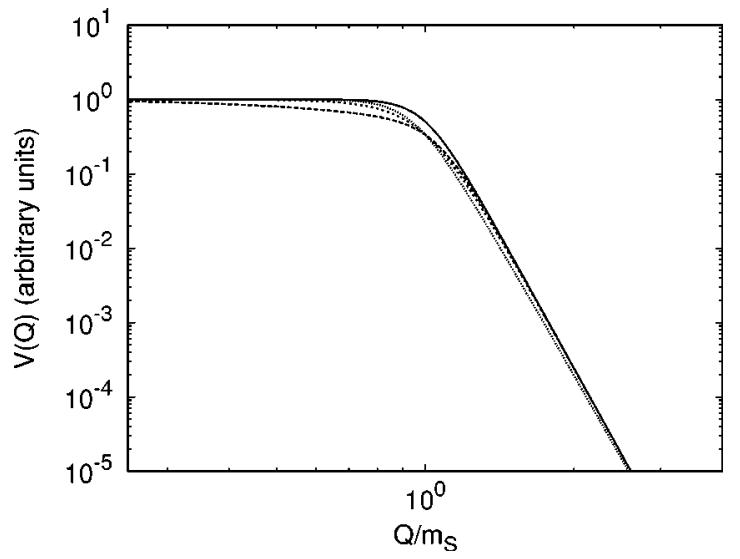

FIG. 3. Different examples of potentials given by Eq. (33). The four curves represent potentials $V(Q)=\left|F_{Y}\right|^{2} /\left[1+\left(Q / m_{S}\right)^{p_{\max }}\right]$ (solid line) and $V(Q)=\left|F_{Y}\right|^{2} /\left[1+\left(Q / m_{S}\right)^{p_{\min }}+\left(Q / m_{S}\right)^{p_{\max }}\right]$, with $p_{\max }=12$ and $p_{\min }=2$ (long-dashed line), $p_{\min }=6$ (short-dashed line), and $p_{\min }=10$ (dotted line).

where $\hat{K}$ parametrizes the rest of the expansion (but of course needs not to be equal to the one introduced previously). This expression should be compared with Eqs. (18) and (19). This time the term $|Y|^{2}$ is present, since we have not assumed anything about the series defining the Kähler potential. The key point is that we have only included terms sensitive to the string scale and not the Planck scale because, in the limit of global SUSY, this one is sent to infinity and therefore the corresponding terms vanish. We have only assumed that the series can be expressed as a polynomial. If this is not the case then a whole knowledge of nonperturbative string theory is required. However, truncating the whole series at the order $p_{\max }$ would require a dynamical explanation which cannot be provided unless in a particular model. For this reason we will study the dependence of the physical observables on the degree of the polynomial.

Let us calculate the corresponding scalar potential (assuming that the quintessence field is real). The only term coming from the Kähler function which gives a contribution to the potential is given by

$$
K^{\bar{Y} Y}=\frac{1}{1+\sum_{p=1}^{p_{\max }} Q^{2 p} / m_{S}^{2 p}},
$$

from which we deduce that

$$
V(Q)=\frac{\left|F_{Y}\right|^{2}}{1+\sum_{p=1}^{p_{\max }} Q^{2 p} / m_{S}^{2 p}} .
$$

Let us study this class of potentials in more detail. Typically, they have the shape represented in Fig. 3. Whatever the precise form of the series, for values of the field such that $Q$ $\ll m_{S}$ the potential is almost flat since the constant term 1 dominates in Eq. (33). This means that we no longer have a divergence of the potential at small $Q$. When the field be- comes of the order of the string scale, $Q \simeq m_{S}$, the precise form of the series matters. But this is true only in a limited region, and one expects that this will not affect the global behavior of quintessence. In the region where $Q \gg m_{S}$, only the term $\left(Q / m_{S}\right)^{2 p_{\max }}$ is important, and the potential reduces to

$$
V(Q) \simeq \frac{\left|F_{Y}\right|^{2} m_{S}^{2 p_{\max }}}{Q^{2 p_{\max }}},
$$

i.e., we recover a Ratra-Peebles potential, and again the detailed form of the series does not matter. This region corresponds to the straight line in Fig. 3 (the slope of which is -12 , since $p_{\max }=6$ was chosen for this plot). Since the tracking behavior essentially depends on the behavior of the field at late times, i.e., before it reaches the Planck mass, the attractor properties of the standard Ratra-Peebles potential still hold in this case, as we have checked numerically. Therefore, the modifications in the potential at low $Q$ do not matter as long as $m_{S}$ is not too large (typically, $m_{S}$ must be $\sim 2$ orders of magnitude smaller than the Planck mass; this bound is even relaxed for high values of $\alpha$ ). Such a behavior was already remarked upon in another context when we considered the quantum corrections to the Ratra-Peebles potential [21]. Note, however, the explicit dependence on the degree $p_{\max }$. This has important observable consequences. Indeed, it appears that potential (34) leads to an equation of state $\omega_{0}$ which exhibits a strong dependence on $p_{\max }$. This is less true for the derivative of the equation of state $\omega_{1}$, as can be seen from Fig. 2 (dashed line). However we shall see that this problem is far less serious when SUGRA corrections are included, in which case the values of $\left(\omega_{0}, \omega_{1}\right)$ accumulate numerically around $(-0.8,0.45)$ in the large $p_{\max }$ regime (Fig. 2, dotted line). This is an interesting indication that the physical observables are stable with respect to variations of the truncation degree.

Another important consequence is that the SUSY breaking scale is now given by

$$
\left|F_{Y}\right|^{2} \simeq \rho_{\mathrm{c}}\left(\frac{m_{P l}}{m_{S}}\right)^{\alpha}
$$

where $\alpha \equiv 2 p_{\max }$. For $m_{P l}=m_{S}$ one recovers the usual result given in Eq. (20). However, the important point is that in the present framework, $m_{P l}$ and $m_{S}$ do not need to be the same, which has the important consequence that now the SUSY breaking scale in the quintessence sector decouples from the critical energy density. Let us show a few orders of magnitude. In particular, one would like to fix the SUSY breaking scale in the quintessence sector to the same value as the SUSY breaking scale in the broken sector, i.e., $\left\langle F_{P}\right\rangle$ $\simeq 10^{20} \mathrm{GeV}$. This would be one step toward an identification between the quintessence sector and the broken sector, thus leaving only one hidden sector. This strategy will be pursued in Sec. V. Fixing $\left|F_{Y}\right| \simeq 10^{20} \mathrm{GeV}^{2}$ and writing $m_{S}$ $=10^{-x} m_{P l}$, we find that $x \simeq 67 / \alpha$. We see that the string scale varies between the $\mathrm{TeV}$ scale and the Planck mass for $\alpha>3$. It is auspicious that to maintain a low value of the 
SUSY breaking scale in the quintessence sector in the large $\alpha$ limit, we need to take values of $m_{S}$ which are closer and closer to the Planck scale. As already stated, as long as $m_{S}$ is a few orders of magnitude smaller than the Planck mass, this has no significant influence of the evolution of the quintessence field today. The previous results follow from the direct coupling between one field $Q$ and the SUSY breaking field $Y$, and does not require any fine-tuning. In particular, the presence of an inverse power law only requires that one can trust the perturbative expansion of the Lagrangian, i.e., one does not need to know the whole power series.

We now need to take into account the SUGRA corrections. As in Sec. III, the form of the potential is given by the positive definite expression $V=e^{\kappa K} K^{Y \bar{Y}}\left\langle\partial_{Y} W\right\rangle^{2}$, where again we have used the fact that the $D$ terms are not modified in SUGRA. The Kähler potential evaluated at the minimum of the potential for the matter fields is a series in $1 / m_{S}$, and reads

$$
K\left(Q,\left\langle\varphi^{i k}\right\rangle\right)=\sum_{n=1}^{n_{\max }} \frac{c_{2 n}}{m_{S}^{2(n-1)}}(Q \bar{Q})^{n},
$$

where we have fixed the other hidden sector fields to their vacuum expectation values. This equation is similar to Eq. (23). Note, however, that we have only kept the dominant $1 / m_{S}$ terms. If $m_{S} \simeq m_{P l}$ we only need to substitute $m_{P l}$ for $m_{S}$ in the expansion. The kinetic term of $Q$ is not normalized. To deal with this problem, as previously, we define a new scalar field according to Eq. (24) (of course, now, the function $f$ needs not to be the same). This leads to the potential

$$
V(\widetilde{Q})=e^{\kappa K\left[f^{-1}(\tilde{Q})\right]} \frac{\left\langle\partial_{Y} W\right\rangle^{2}}{1+\sum_{p=1}^{p_{\max }}\left[f^{-1}(\widetilde{Q})\right]^{2 p} / m_{S}^{2 p}} .
$$

The previous equation gives the generic prediction for any theory which can be effectively described by SUGRA with two scales. Note that taking $m_{P l} \rightarrow \infty$, this reduces to the globally supersymmetric result, as expected. Now we can deduce the form of the potential in the three different regimes, and study how it is affected by the particular form of the theory. First, we note that it does not depend on the superpotential: it is sufficient to have $\left\langle\partial_{Y} W\right\rangle \neq 0$, i.e., a Polonyi field in the quintessence sector. When $Q \ll m_{S}$ then $\widetilde{Q}$ $=\sqrt{2} Q$ and $V(\widetilde{Q}) \simeq\left\langle\partial_{Y} W\right\rangle^{2}$. The potential no longer blows up. In this regime, it does not depend on the details of series (31) or (36). For $Q \simeq m_{S}$ all the terms in the expansion play a role, and the precise shape of the potential cannot be determined unless a specific model is given. But again we expect that we will not affect the cosmological observables since they are determined in a regime where $Q=m_{P l} \gg m_{S}$. For large $Q$ the highest power is only required. As we are interested in the $Q \simeq m_{P l}$ regime we conclude that

$$
K_{Q \bar{Q}}=n_{\max }^{2} c_{2 n_{\max }} \frac{(Q \bar{Q})^{n_{\max }-1}}{m_{S}^{2\left(n_{\max }-1\right)}}
$$

$$
\Rightarrow \widetilde{Q}=\sqrt{2 c_{2 n_{\max }}} \frac{Q^{n_{\max }}}{m_{S}^{n_{\max }-1}},
$$

leading to

$$
V(\widetilde{Q})=A e^{\kappa\left(\widetilde{Q}^{2} / 2\right)} \frac{\left\langle\partial_{Y} W\right\rangle^{2}}{\widetilde{Q}^{2 p_{\max } / n_{\max }},}
$$


arrange themselves such that $\kappa \widetilde{Q}^{2} / 2$ appears in the potential, without any additional multiplicative factor in the argument of the exponential. We can identify $\alpha \equiv 2 p_{\max } / n_{\max }$. Therefore, in this regime, we recover the SUGRA quintessence potential which now appears as a generic property of any effective SUGRA theory with two scales. Now the degrees of the truncated series $n_{\max }$ and $p_{\max }$ play competing roles. In particular, three natural behaviors can occur. In the first one $\alpha$ goes to zero. This is physically disfavored, as this would require that $\left\langle\partial_{Y} W\right\rangle^{2}$ converge to the critical energy density $\rho_{c}$. Similarly $\alpha$ can go to infinity, with the need for $m_{S}$ to be closer and closer to the Planck scale. Finally, $\alpha$ can remain finite. In this case we do not need to fine-tune the SUSY breaking scale. The point is that the observables do not depend very much on $\alpha \equiv 2 p_{\max } / n_{\max }$. Indeed, a large range of values of $\alpha$ lead to the same CMB spectrum and the same dependence of the equation of state at small redshifts (see Fig. 2, dotted line, and Ref. [32]). It is remarkable that from an a priori very complicated theory, we end up with the conclusion that observables like $\left(\omega_{0}, \omega_{1}\right)$ are uniquely determined by potential (40). Since typically, we expect that the coefficient $c_{n_{\text {max }}}$ is of order 1 , we deduce that the SUSY breaking scale is again given by relation (35). In order to justify that the previous considerations really lead to a successful and realistic model for quintessence, we need to study the process of SUSY breaking in more detail.

\section{SUPERSYMMETRY BREAKING}

In the previous sections we have seen that it is necessary to assume three different sectors, two of them being hidden. In this section, we thoroughly analyze the consequences of SUSY breaking, both from cosmological and particle physics points of view.

\section{A. Spontaneous vs explicit supersymmetry breaking}

A first study of SUSY breaking in the context of quintessence was made in an interesting paper by Kolda and Lyth [35]. There, the authors pinpointed a possible incompatibility between quintessence and SUSY. Indeed the expansion of Eq. (5) comprises the two terms

$$
K_{Y \bar{Y}}\left|F^{Y}\right|^{2}+W_{Y} F^{Y}+\bar{W}_{\bar{Y}} F^{\bar{Y}} .
$$

Assuming that SUSY is broken explicitly by $F_{Y}$ leads to a polynomial expansion of the scalar potential in $Q$ when using the general Taylor expansion of $K_{Y \bar{Y}}$. Fortunately, in SUGRA one must consider SUSY as a local gauge theory wich cannot be broken explicitly, as the electroweak symme- 
try which is not broken by putting an explicit gauge symmetry breaking mass in the Lagrangian. SUSY is broken spontaneously by the nonvanishing vev of $F$ terms obtained by solving the equations of motion. This leads to a super-Higgs mechanism, where the would-be massless Goldstone fermion is eaten by the gravitino which becomes massive [37]. As the $F$ terms are auxiliary field terms with no kinetic terms, one can solve Eq. (41) algebraically to give

$$
F^{Y}=-K^{Y \bar{Y}} \bar{W}_{\bar{Y}}
$$

leading to the potential investigated in the previous sections. It is apt that an intrinsic feature of SUGRA prevents this type of quintessential difficulty.

\section{B. Moduli supersymmetry breaking}

We have seen that a quintessence potential can be obtained in a hidden (quintessence) sector. On the other hand, we have assumed that SUSY was broken in another hidden sector. Therefore, one may wonder whether it would not be possible to consider only one hidden sector where SUSY is broken and, at the same time, to which the quintessence field belongs. In this section, we will include the effects due to other $F$ terms, and study the modifications that they impose on the potential. In particular we suppose that these are the single Kähler moduli $T$ and the dilaton field $S$ where the superfields $T$ and $S$ belong to the unique (postulated) "broken-quintessence" hidden sector. Because of SUSY breaking the potential will have the form

$$
\begin{aligned}
V_{\text {bro }}(Q)= & V(Q)+e^{\kappa K}\left(K^{T \bar{T}}\left|F_{T}\right|^{2}+K^{S \bar{S}}\left|F_{S}\right|^{2}\right)+|D|^{2} \\
& -3 m_{3 / 2}^{2} / \kappa+V_{\text {add }},
\end{aligned}
$$

where the potential $V(Q)$ is the quintessence potential obtained previously. The $D$ terms are independent of $Q$, as this is a neutral field. The gravitino mass $m_{3 / 2}$ is nonzero due to the breaking of SUSY. The last term $V_{\text {add }}$ springs from the visible sector, and gives large contributions to the cosmological constant. This is the cosmological constant problem: $V_{\text {bro }}(Q)$ contains huge constant terms which, a priori, dominate all the other contributions. The $F_{S}$ and $F_{T}$ auxiliary fields are given by

$$
F_{S, T}=-\partial_{S, T} W-\kappa\left(\partial_{S, T} K\right) W
$$

and depend on the nonperturbative corrections to the superpotential which are responsible for the breaking of SUSY. There is a strong dependence of $F_{T}$ and $F_{S}$ on the Kähler potential. To go further we need to return to Eq. (31), and to be more specific about the forms of the function $\hat{K}$. We take a generic form of the Kähler function as

$$
\begin{aligned}
\hat{K}= & \frac{1}{\kappa}[-3 \ln (T+\bar{T})-\ln (S+\bar{S})] \\
& +m_{S}^{2} \sum_{p q k} d_{p q k}(S+\bar{S})^{-p}(T+\bar{T})^{-q}\left(\frac{Q \bar{Q}}{m_{S}^{2}}\right)^{k},
\end{aligned}
$$

where we assume that this is a polynomial in $Q \bar{Q}$ (the coefficients $d_{p q k}$ are just the coefficients of the polynomial). Only inverse powers of $m_{S}$ were taken into account, as order by order in $Q$ the inverse powers of $m_{P l}$ are suppressed. Computing the derivative with respect to $S$ and $T$ leads to a polynomial dependence on $Q$ of $F_{S}$ and $F_{T}$. This implies that the SUSY breaking scale varies during the evolution of the universe, and therefore the sparticle masses become strongly time dependent. Indeed, the mass matrix of the scalars depends explicitly on the $F$ terms,

$$
m_{A \bar{B}}^{2}=e^{\kappa K}\left(\frac{\kappa}{3} K_{A \bar{B}} K_{C \bar{D}}-R_{A \bar{B} C \bar{D}}\right) F^{C} \bar{F}^{\bar{D}},
$$

where the second term involves the Riemann tensor deduced from the Kähler potential. It is easy to see that a polynomial dependence on $Q$ for $F_{S}$ and $F_{T}$ leads to a polynomial dependence on $Q$ of the masses from $K^{T \bar{T}_{T}} F_{T} \bar{F}_{\bar{T}}$ and $K^{S \bar{S}_{F}} F_{S} \bar{F}_{\bar{S}}$. At large $Q$ this behaves like $\left(Q / m_{\mathrm{S}}\right)^{2 k_{\max }}$, where $k_{\max }$ is the dominant term in Eq. (45).

To avoid this we must conclude that the quintessence field decouples from the SUSY breaking sector:

$$
d_{p q k}=0, \quad k \neq 0 .
$$

On the whole we find that the SUSY breaking sector and the quintessence sector must be separate.

Coming back to Eq. (43), there is a negative contribution from the gravitino mass:

$$
m_{3 / 2}=\kappa e^{\kappa K / 2}\langle W\rangle .
$$

Combining with the $F_{S}$ and $F_{T}$ terms, this leads to the following term in the potential $V_{\text {bro }}$ :

$$
e^{\kappa K}\left(K^{T \bar{T}}\left|F_{T}\right|^{2}+K^{S \bar{S}}\left|F_{S}\right|^{2}-3 \kappa\langle W\rangle^{2}\right) .
$$

In the early Universe this is a cosmological constant, as the term in brackets is a constant. As $Q$ increases the exponential corrections become relevant. So this term acts as a slowly varying cosmological constant. Moreover, we can expect a large contribution $V_{\text {add }} \simeq m_{W}^{4}$ from the visible sector. Both contributions should be large compared to the critical density of the Universe. Nevertheless there is a strong constraint springing from the existence of an attractor. The attractor condition [Eq. (2)] should be compatible with the requirement that the total potential reproduces $\Omega_{\Lambda} \rho_{c}$. It can easily be seen that, if the slowly varying and constant contributions are much larger than the critical density, then the attractor disappears. Consequently we shall assume that the extra constant and slowly varying pieces in the potential vanish altogether. This is another manifestation of the fact that it is necessary to assume that the cosmological constant problem is solved before considering the quintessence hypothesis. In the context of quintessence, the relevant question is whether the dynamical part of the potential after SUSY breaking is modified. In particular, this leads to the requirement that the contributions from the visible sector and the broken sector must vanish independently, i.e., 


$$
V_{\text {add }}=0, \quad K^{T \bar{T}}\left|F_{T}\right|^{2}+K^{S \bar{S}}\left|F_{S}\right|^{2}=3 \kappa\langle W\rangle^{2} .
$$

The second of these constraints is the usual fine-tuning of the SUSY breaking sector.

Let us now consider the contribution to the scalar masses due to the Polonyi field $Y$,

$$
\left(\frac{\kappa}{3} K_{A \bar{B}}-R_{A \bar{B} Y \bar{Y}} K^{Y \bar{Y}}\right) V(Q),
$$

which is negligible now due to the smallness of $V(Q)$. The scalars receive a mass from the $F_{S}$ and $F_{T}$ terms, which reads $[38,39]$

$$
m_{A \bar{B}}^{2}=m_{3 / 2}^{2} K_{A \bar{B}}-e^{\kappa K}\left(R_{A \bar{B} S \bar{S}} F^{S} \bar{F}^{\bar{S}}+R_{A \bar{B} T \bar{T}} F^{T} \bar{F}^{\bar{T}}\right) .
$$

Note that the sparticle masses will have a universal redshift dependence coming from exponential factor in Eq. (48). This dependence is only relevant in the recent past. It would be interesting to study the associated phenomenology. There is a final constraint springing from the gauginos masses $[38,39]$,

$$
m_{a}=\frac{\sqrt{\kappa}}{2} e^{\kappa K} F^{I} \partial_{I} \ln g_{a}^{-2},
$$

where $g_{a}$ is the gauge coupling of the $a$ th gauge group. To leading order one can expand

$$
g_{a}^{-2}=S+\bar{S}+\beta \sqrt{\kappa}(Y+\bar{Y}),
$$

where we have included a dependence on $Y$. This is what happens in type I models if the Polonyi field can be identified with the blowing up moduli. Nevertheless the presence of $K^{Y \bar{Y}}$ implies that the $F_{Y}$ contribution is negligible. So we find that that the masses of sparticles do not depend on $F_{Y}$. This allows for independent supersymmetry mechanisms in the "broken" and "quintessence" sectors. In particular the mechanism of Sec. III, where

$$
F_{Y}^{2}=\left\langle Z^{2}\right\rangle^{2}=\left(\frac{m_{P l}}{m_{S}}\right)^{\alpha} \rho_{c}
$$

is viable. Phenomenologically we should impose that the corresponding Fayet-Iliopoulos term is larger than the weak scale. This leads to

$$
\frac{m_{S}}{m_{P l}} \leqslant\left(\frac{\rho_{c}}{m_{W}^{4}}\right)^{1 / \alpha},
$$

which is reasonable as soon as $\alpha>3$. We can even go further by noticing that the Fayet-Iliopoulos term is of the order of the string scale. Imposing that $F_{Y}=m_{S}^{2}$ leads to

$$
\rho_{\mathrm{c}}=\frac{m_{\mathrm{S}}^{4+\alpha}}{m_{P l}^{\alpha}} .
$$

In new type I string scenarios the string scale can be as low as the TeV region. In that case this leads to $\alpha=4$. This determines $p_{\max }=2$ for a flat Kähler potential in $Q$. The relation

$$
\rho_{\mathrm{c}}^{1 / 4}=\frac{m_{S}^{2}}{m_{P l}}
$$

was advocated in Ref. [40] to obtain a natural solution to the coincidence problem. We find that it can be embedded in a SUGRA description of quintessence with two scales.

In conclusion we have seen that quintessence is compatible with SUSY breaking, and should belong to a hidden sector different from the hidden broken sector.

\section{INFLUENCE OF A MINIMUM IN THE QUINTESSENCE POTENTIAL}

From the above considerations, it seems that a generic consequence of taking into account high energy physics is the presence of a minimum in the quintessence potential. This differs from the Ratra-Peebles case, where the potential is monotonic and goes to zero at infinity. Therefore, one may wonder what the physical consequences of the presence of this minimum are. The purpose of this section is to study this question.

\section{A. Oscillations of the quintessence field}

The SUGRA potential possesses a minimum located at $Q_{\min }=\sqrt{\alpha} / \kappa^{1 / 2}=O\left(m_{P l}\right)$; see Fig. 1. Thus, a priori, this could modify the final evolution of the field. Therefore, let us expand the field around the minimum; we write

$$
\bar{Q}=\sqrt{\alpha}+\bar{q},
$$

where $\bar{Q} \equiv \kappa^{1 / 2} Q$ is dimensionless and where $\bar{q}$ is a small quantity. If we neglect the quadratic order, the Einstein equation reads $H^{2}=H_{0}^{2}=(\kappa / 3) V(\sqrt{\alpha})$ which implies that $a(t)$ $=a_{0} e^{H t}$. On the other hand, the Klein-Gordon equation is given by

$$
\ddot{\bar{q}}+3 H_{0} \dot{\bar{q}}+6 H_{0}^{2} \bar{q}=0 .
$$

The solution to this equation is given by the following expression:

$$
\bar{q}(t) \propto \exp \left[\left(-\frac{3}{2} \pm i \frac{\sqrt{15}}{2}\right) H_{0} t\right]
$$

This solution is oscillatory with a damping term proportional to $a^{-3 / 2}$. The period of the oscillations is equal to $\simeq H_{0}^{-1}$, i.e., is equal to the age of the Universe today. Therefore, it is clear that no oscillation took place until now since the age of the Universe is the time already necessary to reach the region where the oscillations could occur. Conversely, the future of the Universe will be different in comparison with the RatraPeebles potential case. Numerically, for the case $\alpha=11$, the redshifts at which the field stops are $z=-0.65,-0.92$, -0.98 , etc. The first redshift corresponds to $a / a_{0} \sim 2.85$, i.e., 


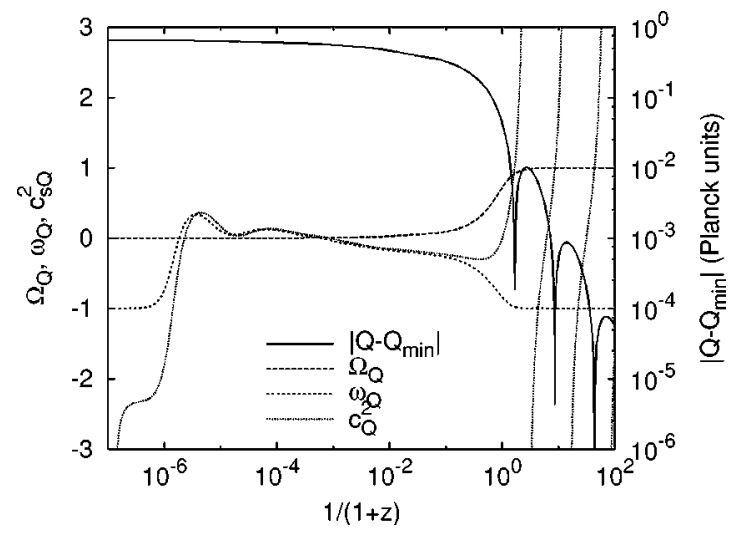

FIG. 4. Overall evolution of the quintessence field. We start at a redshift of $z=10^{7}$ with a quintessence field initially at rest $\left(\omega_{Q} \rightarrow\right.$ -1 , short-dashed line) and subdominant $\left(\Omega_{Q} \rightarrow 0\right.$, long-dashed line). Then the field joins the attractor around $z \simeq 10^{4}$. It remains on this attractor as long as it is subdominant, i.e., $\Omega_{Q} \ll 1$. When it starts to dominate, it gradually behaves as a cosmological constant ( $\omega_{Q} \rightarrow-1$ again around $z \simeq 0$ ). Then the field experiences some damped oscillations around its minimum (solid line). The behavior of the parameter $\omega_{Q}$ can be studied by looking at the variable $c_{Q}^{2}$ $\equiv \dot{p}_{Q} / \dot{\rho}_{Q}$ (dotted line), which diverges when $\omega_{Q}$ reaches -1 [this occurs initially and when $Q(t)$ reaches an extremum].

to a time where the scale factor is 2.85 larger than today (see Fig. 4). This is of course independent of the initial conditions provided that we are initially in the allowed range.

It is of course possible that some oscillations occur before today, but this is not easy. The main reason is that the quintessence field rolls rather slowly toward the bottom of its potential, so that the quintessence density parameter $\Omega_{Q}$ is almost equal to 1 at the time where the field stops for the first time (as can be seen in Fig. 4). Another possibility is that $\Omega_{Q}$ is of order unity at early time. In this case, the field is initially very small, and correspondingly its energy density is large. Then, the field is in a "fast-roll" regime, i.e., $\omega_{Q} \simeq 1$, and is not slowed down enough by the expansion. It then goes through (still in a fast-roll regime) its minimum, and is stopped by the very steep exponential growth of the potential at large $Q$. Such a behavior does not affect the behavior of

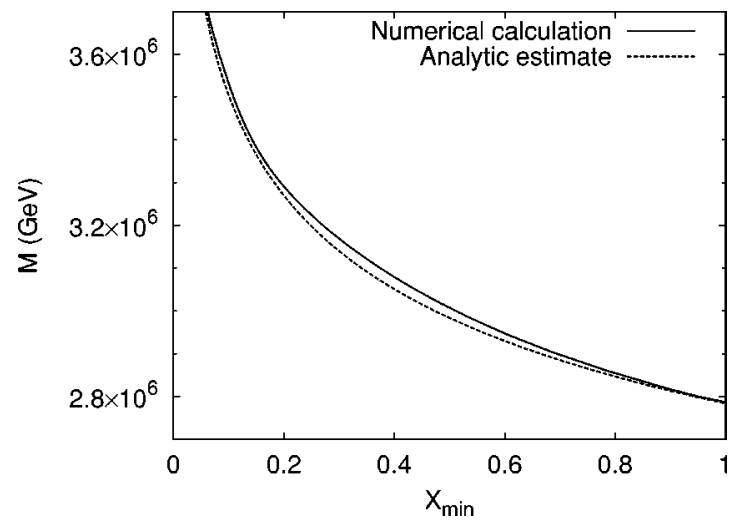

FIG. 5. Dependence of the mass scale $M$ with the minimum of the potential $X_{\min }$. The numerical computation gives a very good agreement with the estimate of Eq. (63).

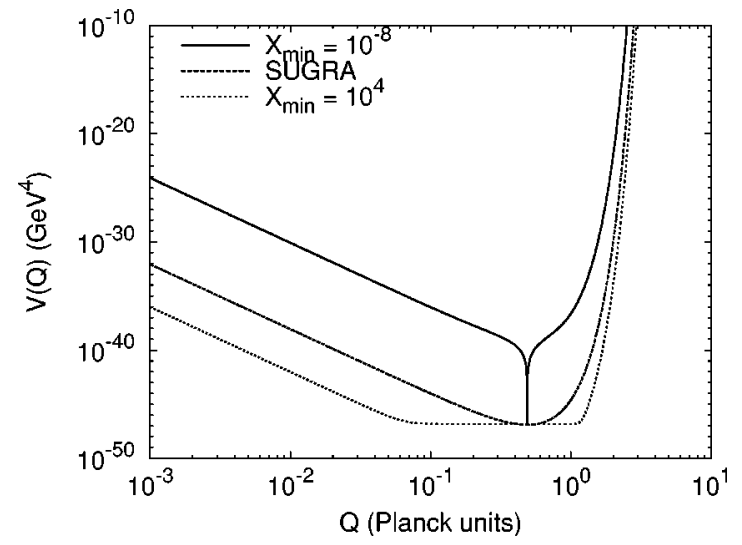

FIG. 6. Shape of the quintessence potential for various values of $X_{\min }$. The potentials were normalized so that $\Omega_{Q}=0.7$ today, which roughly corresponds to requiring that the minimum of the potential is equal to the critical density today. Note the presence of a broad, flat region for high values of $X_{\min }$, and a deep and narrow depression for small values of $X_{\min }$. These features have a large importance on the evolution of the quintessence field today.

the quintessence field today, but can leave some imprints in the high frequency part of the primordial gravitational wave spectrum (see, e.g., Ref. [36]).

\section{B. Amplitude of the minimum}

Let us now study the influence of a pure cosmological constant term in the quintessence potential. We would like to know whether we can change the value of the minimum and, in particular, whether it is possible to put it to zero. Therefore, we take a SUGRA potential to which we add a constant term

$$
V(Q)=e^{\kappa Q^{2} / 2} \frac{M^{4+\alpha}}{Q^{\alpha}}+\left(X_{\min }-1\right) V_{\min }
$$

In this expression $V_{\min }$ is the value of the potential at its minimum, i.e., for $Q=\sqrt{\alpha / \kappa}$ and $X_{\min } \geqslant 0$ a free parameter. $X_{\min }=0$ corresponds to a vanishing minimum, and $X_{\min }=1$ reduces the above potential to the standard SUGRA potential. We would like to emphasize that there is no fine-tuning of the location of the minimum; it follows directly from the shape of the potential (and is of course independent of the constant $M$ ). The fact that the field is today near the minimum of the potential follows directly from the fact that, because of the presence of the attractor, the field is today of the order of the Planck mass, which also turns out to be the location of the minimum of the potential. Again, no finetuning is required to have this property which arises naturally in SUGRA quintessence.

Let us start with the case where the minimum is not zero. The presence of a constant term can influence the shape of the potential and the value of the constant $M$, as explained below. Let us start with the constant $M$. In all the cases presented here, as mentioned above, the constant $M$ is found numerically by requiring that $\Omega_{Q}=0.7$ today. In all the cases of interest, the quintessence equation of state is such that 


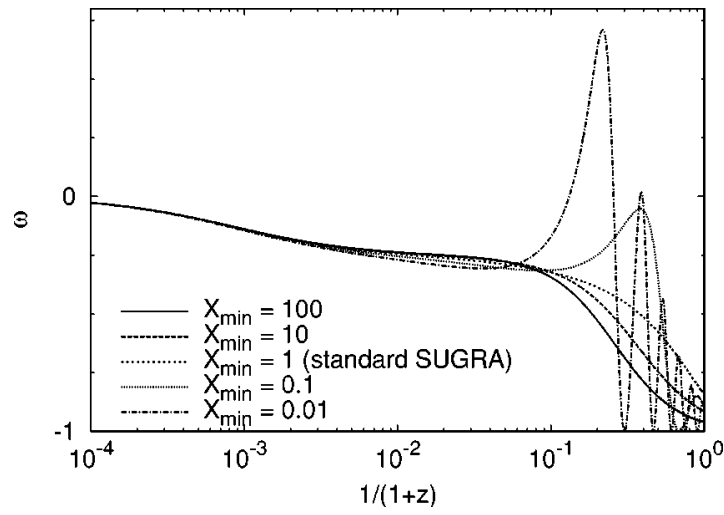

FIG. 7. Evolution of the quintessence field equation of state parameter for several values of $X_{\min }$. The field starts at a moderately high redshift $(z \simeq 100)$ from its attractor value (which means $\omega_{Q} \simeq-0.25$ for $\alpha=6$ here), and subsequently starts to behave as a cosmological constant as its energy density dominates (we have taken $\Omega_{Q}=0.7$ today). As explained in the text, large values of $X_{\min }$ all lead to essentially the same behavior, whereas low values of $X_{\min }$ cause the field to oscillate.

$-1 \leqslant \omega_{Q} \leqslant 0$ today. This means that a significant part of the energy density of the field is determined by its potential energy. In presence of the additional constant term, this implies that the constant $M$ is no longer given by Eq. (3), but rather by

$$
M \simeq\left(\frac{\rho_{c} m_{P l}^{\alpha}}{X_{\min }}\right)^{1 /(4+\alpha)}
$$

This is what we can check on Fig. 5. Knowing how to determine $M$, we can now turn to the shape of the potential. For large values of $X_{\min }$, there is a large region where the potential is almost flat. This means that when the quintessence field enters this region, it behaves very quickly as a cosmological constant. Conversely, small values of $X_{\min }$ produce a deep and narrow "hole" in the potential in which the field

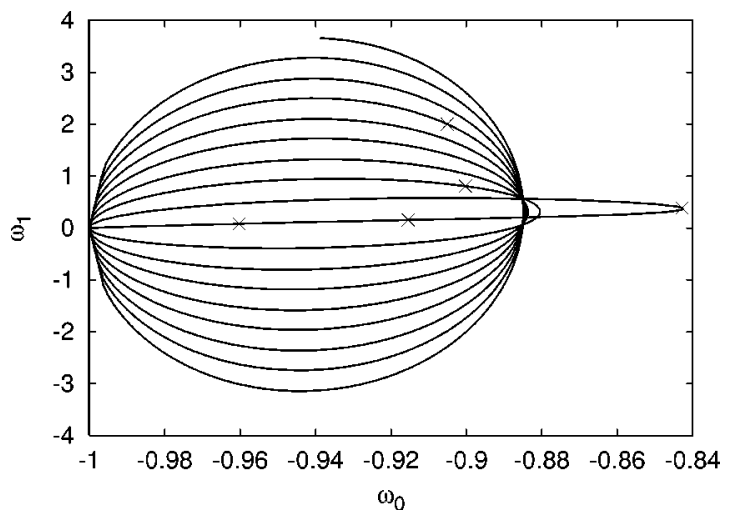

FIG. 8. Today's value of the quintessence equation of state parameter $\omega_{0}$ and its derivative $\omega_{1}$ for several values of $X_{\min }$. The crosses represent the values of $X_{\min }$ used in Fig. 7 (same color code). Note that for low values of $X_{\min }$, the oscillations of the field are quite rapid, and therefore, the equation of state parameter is not very well approximated by $\omega \sim \omega_{0}+z \omega_{1}$, even for a relatively short interval of the redshift.

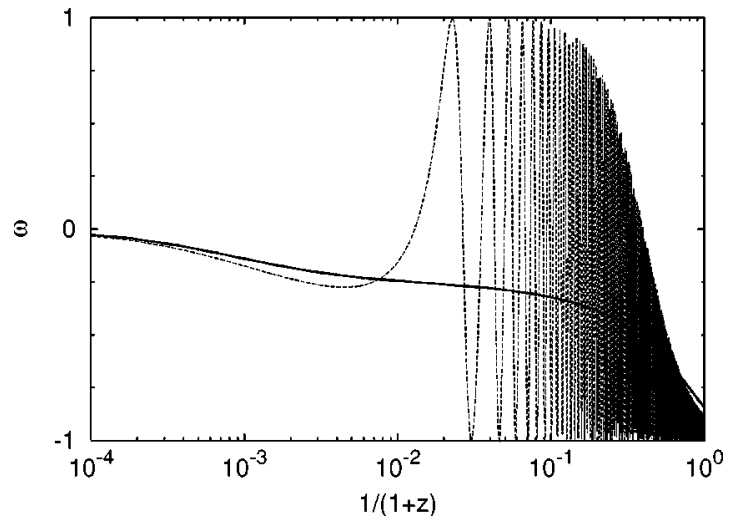

FIG. 9. Evolution of the equation of state parameter $\omega_{Q}$ as a function of the redshift for $X_{\min }=1$ (solid) and $X_{\min }=10^{-5}$ (dashed). For such a low value of $X_{\min }$, only for a small redshift can one see the decay of $\omega_{Q}$ toward -1 , as indicated by the decreasing envelope of the curve.

oscillates when it falls into it. These two cases are represented in Fig. 6. The addition of a constant term must have some observable consequences today. This is what we can check in Fig. 7, where we plot the value of $\omega_{Q}$ as a function of the redshift. As expected, large values of $X_{\min }$ do not significantly differ from the $X_{\min }=1$ case, except that the equation of state parameter $\omega$ goes faster to -1 (the potential is less steep). Conversely, the oscillations for small values of $X_{\min }$ are clearly observable. This is due to the fact that in this case, Eq. (60) reads

$$
\ddot{\bar{q}}+3 H_{0} \dot{\bar{q}}+6 \frac{H_{0}^{2}}{X_{\min }} \bar{q}=0,
$$

so that the frequency of the oscillations can be arbitrarily large. Then if we plot the values of $\left(\omega_{0}, \omega_{1}\right)$ for several values of $X_{\min }$, the oscillations of the field translate into ellipses in the $\left(\omega_{0}, \omega_{1}\right)$ plane; see Fig. 8.

Finally, we would like to stress some important properties of the dynamic of the quintessence field in the case of a vanishing value of $X_{\min }$, i.e., when one tries to set the potential to zero. A decreasing value of $X_{\min }$ leads to an increasing number of oscillations experienced by the quintessence field before today; see Eq. (64). Numerically, this translates into a very weird behavior of the function $\omega_{Q}(z)$ as $X_{\min }$ goes to 0 ; see Fig. 9. Now, in the vicinity of the minimum of the potential, the potential has a quadratic shape. Therefore, this leads to an equipartition between the kinetic energy and the potential energy, and therefore to an average equation of state parameter $\omega_{Q}$ equal to 0 , a wellknown behavior of the inflaton field at the end of inflation $[41] .^{2}$ In this case, the equation of state of the field is exactly the same as the one of ordinary matter. As a consequence, the ratio between $\Omega_{Q}$ and $\Omega_{m}$ becomes a constant. This means that

\footnotetext{
${ }^{2}$ This point can in principle be evaded if we suppose that the potential behaves like $\left(Q-Q_{\min }\right)^{\beta}$, with $0<\beta<2$, but this seems to be an unlikely possibility in the case presented here.
} 
the value of $\Omega_{Q}$ today is approximatively given by the value of $\Omega_{Q}$ when the field started its oscillations, denoted $\Omega_{Q}^{\text {osc }}$ in what follows. Then, the relevant question is: can $\Omega_{Q}^{\text {osc }}$ be equal to (say) 0.7 ? The answer to this question depends on the physical reason which causes the field to leave the attractor. A priori, two situations can be envisaged. First, the field leaves the attractor because it has not yet reached (or felt) the minimum and it starts to dominate. This is what happens in the Ratra-Peebles case (for which there is no minimum). Second, conversely, it has not yet started to dominate but the field "feels" the presence of a minimum. In the second case, by definition we have $\Omega_{Q}^{\text {osc }} \ll 1$, and the answer to the question above is "no." Therefore, only the first situation remains a possibility. Let us study this situation in more detail. In particular, one may wonder whether it can really happen that the field dominates before encountering the minimum. The field dominates when $Q=Q_{\text {end }}$, defined by the condition $\rho_{Q} \simeq \rho_{m} / x$, where $x$ is an arbitrary number. A reasonable value for $x$ is, for example, $x=10$. Using the equation of the attractor [see Eq. (2)], it is easy to establish that $\kappa Q_{\text {end }}^{2}$ $=\alpha(\alpha+2) /[3(x+1)]$. On the other hand, we have $\kappa Q_{\min }^{2}$ $=\alpha$. Therefore if $\alpha>3(x+1)-2$, then $Q_{\text {end }}<Q_{\min }$, and we are in the desired situation. However, this is not so simple, because the width of the hole, denoted here as $\delta\left(\kappa Q^{2}\right)$, matters. We are in a good position only if $\kappa\left(Q_{\min }^{2}-Q_{\text {end }}^{2}\right)$ $>\delta\left(\kappa Q^{2}\right)$; otherwise we cannot say that the field does not feel the minimum of its potential. It is not totally trivial to calculate the width of the potential, which is not symmetric with respect to $Q_{\min }$. A fair estimate is given by the difference between $Q_{\min }$ and the value of $Q$, such that the SUGRA potential becomes different from the Ratra-Peebles (RP) potential, i.e., for $Q$ such that $\left|V_{\text {SUGRA }}(Q) / V_{\mathrm{RP}}(Q)\right| \simeq y$, where $y$ is an arbitrary number (for example, $y=0.1$ ). This gives a width equal to $\delta\left(\kappa Q^{2}\right)=\alpha-2 \ln (y+1)$. Of course, the comparison depends on the precise values of $x$ and $y$, but for reasonable values one reaches the conclusion that the width of the potential is always of the same order of magnitude as the difference $Q_{\min }-Q_{\text {end }}$. Therefore, even if $Q_{\text {end }}<Q_{\min }$, we will obtain $\Omega_{Q}^{\text {osc }} \ll 1$. As a consequence, the energy density of the quintessence field cannot dominate, and there is no possibility of reaching a value of $\Omega_{Q}=0.7$ today if the minimum is put to zero.

\section{CONCLUSIONS}

In this paper, we have studied the model building problem of quintessence in the context of SUGRA viewed as the low energy limit of string theory. In this context, the theory is described by two scales: the Planck scale and the string scale. A priori, there is no reason to assume that these two scales are equal. If indeed the string scale decouples from the Planck scale, we have shown that the SUGRA quintessence potential arises naturally in this framework. In addition, it was demonstrated that the potential is stable against corrections in the Kähler potential and if SUSY breaking is taken into account. A generic property of the SUGRA quintessence potentials is the presence of a minimum. We have shown that the field today is always close to this minimum. This requires no fine-tuning, and is due to the fact that the minimum turns out to be of the order of the Planck mass, the value that the field has when it leaves the attractor, at small redshifts. We have also demonstrated that the minimum of the potential cannot be put to zero while keeping $\Omega_{Q}$ to a value of the order of the critical energy density today.

\section{ACKNOWLEDGMENTS}

Section VI of this paper was motivated by numerous interesting questions asked by Karim Benabed, Francis Bernardeau, and Pierre Binétruy. It is a pleasure to thank them for very useful exchanges and comments. We also thank Pierre Binétruy and Stephane Lavignac for a careful reading of the manuscript. A.R. is funded by EC-Research Training Network CMBNET (contract number HPRN-CT-200000124).
[1] S. Weinberg, Rev. Mod. Phys. 61, 1 (1989).

[2] Supersymmetry, edited by S. Ferrara (Elsevier, Amsterdam, 1987).

[3] E. Witten, Nucl. Phys. B185, 513 (1981).

[4] J. Polchinski, String Theory (Cambridge University Press, Cambridge, England, 1998).

[5] E. Witten, Lecture given at DM2000, e-print hep-ph/0002297.

[6] S. Perlmutter et al. Nature (London) 391, 51 (1998); S. Perlmutter et al., Astrophys. J. 517, 565 (1999); P. M. Garnavich et al., Astrophys. J. Lett. 493, L53 (1998); A. G. Riess et al., Astron. J. 116, 1009 (1998).

[7] P. de Bernardis et al., Nature (London) 404, 955 (2000).

[8] A. E. Lange et al., Phys. Rev. D 63, 042001 (2001).

[9] S. Hanany et al., Astrophys. J. Lett. 545, L5 (2000).

[10] A. Balbi et al., Astrophys. J. Lett. 545, L1 (2000).

[11] M. Tegmark, Astrophys. J. Lett. 514, L69 (1999).

[12] I. Zehavi and A. Dekel, Nature (London) 401, 252 (1999).
[13] L. Wang, R. R. Caldwell, J. P. Ostriker, and P. J. Steinhardt, Astrophys. J. 530, 17 (2000).

[14] B. Ratra and P. J. E. Peebles, Phys. Rev. D 37, 3406 (1988).

[15] C. Wetterich, Astron. Astrophys. 301, 321 (1995).

[16] P. G. Ferreira and M. Joyce, Phys. Rev. D 58, 023503 (1998).

[17] I. Zlatev, L. Wang, and P. J. Steinhardt, Phys. Rev. Lett. 82, 896 (1999).

[18] P. J. Steinhardt, L. Wang, and I. Zlatev, Phys. Rev. D 59, 123504 (1999).

[19] I. Antoniadis, N. Arkani-Hamed, S. Dimopoulos, and G. Dvali, Phys. Lett. A 436, 257 (1998).

[20] P. Brax and J. Martin, Phys. Lett. B 468, 40 (1999).

[21] P. Brax and J. Martin, Phys. Rev. D 61, 103502 (2000).

[22] E. J. Copeland, N. J. Nunes, and F. Rosati, Phys. Rev. D 62, 123503 (2000).

[23] A. Brignole, L. E. Ibanez, and C. Munoz, Nucl. Phys. B422, 125 (1994). 
[24] L. E. Ibanez, C. Munoz, and S. Rigolin, Nucl. Phys. B553, 43 (1999).

[25] A. Masiero, M. Pietroni, and F. Rosati, Phys. Rev. D 61, 023504 (2000).

[26] J.-P. Uzan, Phys. Rev. D 59, 123510 (1999).

[27] P. Binétruy, Phys. Rev. D 60, 063502 (1999).

[28] M. B. Green and J. H. Schwarz, Phys. Lett. 149B, 117 (1984).

[29] M. Dine, N. Seiberg, and E. Witten, Nucl. Phys. B289, 585 (1987).

[30] G. Polonyi, University of Budapest report.

[31] L. O'Raifeartaigh, Nucl. Phys. B96, 331 (1975).

[32] P. Brax, J. Martin, and A. Riazuelo, Phys. Rev. D 62, 103505 (2000).

[33] Recently, the paper by J. Hwang and H. Noh, astro-ph/0103244, appeared where it was claimed that one standard equation present in all the textbooks and used in Ref. [32] is not correct. We find ourselves in disagreement with such a statement and believe that this equation is correct.

[34] P. Astier, astro-ph/0008306.

[35] C. Kolda and D. Lyth, Phys. Lett. B 458, 197 (1999).

[36] A. Riazuelo and J.-P. Uzan, Phys. Rev. D 62, 083506 (2000).

[37] E. Cremmer, B. Julia, J. Scherk, S. Ferrara, L. Girardello, and P. Nieuwenhuizen, Nucl. Phys. B147, 105 (1979).

[38] S. P. Li, R. Peschanski, and C. A. Savoy, Nucl. Phys. B289, 206 (1987).

[39] V. S. Kaplunovski and J. Louis, Phys. Lett. B 306, 269 (1993).

[40] N. Arkani-Hamed, L. Hall, C. Kolda, and H. Murayama, Phys. Rev. Lett. 85, 4434 (2000).

[41] A. D. Linde, Particle Physics and Inflationary Cosmology (Harwood Academic, New York, 1990). 